\title{
The Effects of Intravenous Vitamin C Administration on hs-CRP and Tumor Necrosis Factor- $\alpha$ Levels in Haemodialysis Patients
}

\section{Hamid Taiebi Khosroshahi ${ }^{1}$, Yashar Talebi ${ }^{2}$, Siamak Ahmadzadeh ${ }^{2}$, Afshin Habibzadeh $^{2}$, Seyyed Ehsan Mousavi ${ }^{2}$, ${ }^{\text {and }}$ Mohammadreza Khalilzadeh $^{3}$}

\author{
${ }^{1}$ Biotechnology Research Center, Tabriz University of Medical Sciences, Tabriz, Iran; \\ ${ }^{2}$ Department of Nephrology, Imam Reza Hospital, Faculty of Medicine, Tabriz University of \\ Medical Sciences, Tabriz, Iran; ${ }^{3}$ Hemodialysis Center, Imam Reza Hospital, Tabriz, Iran
}

Corresponding author: Hamid Taiebi Khosroshahi, MD, Biotechnology Research Center, Tabriz University of Medical Sciences, Tabriz, Iran

Submission date: January 18, 2011; Acceptance date: July 23, 2011; Publication date: August 30,2011

\begin{abstract}
:
Background: It has been proved that pre-inflammatory factors increase mortality and morbidity of cardio-vascular diseases especially in hemodialysis patients. There is a great evidence for the increase of pre-inflammatory factors and vitamin $\mathrm{C}$ deficiency in dialysis patients. Limited data, however, are available regarding the effects of vitamin C supplementation on reduction of inflammatory markers in such patients.
\end{abstract}

Objectives: The aim of this study is to determine the changes of hs-CRP and Tumor Necrosis Factor- $\alpha$ (TNF- $\alpha$ ) levels after administration of vitamin C in End Stage Renal Disease patients (ESRD) on Hemodialysis

Methods and Materials: In this clinical trial, vitamin $\mathrm{C}$ was administered intravenously (IV), (500 mg two times per week) for 2 months in 41 chronic hemodialysis patients and the hs-CRP and TNF- $\alpha$ levels of these patients were compared with a control group with no vitamin $\mathrm{C}$ as treatment. Besides hs-CRP and TNF- $\alpha$, detailed laboratory results were evaluated before and after the study. The statistical analysis was performed using SPSS version 16 at the end of the study.

Results: The hs-CRP and TNF- $\alpha$ levels reduced significantly in hemodialysis patients after vitamin $\mathrm{C}$ supplementation (hs-CRP decreased from 7.27 \pm 3.70 to $6.60 \pm 3.75, \mathrm{P}<0.001$ and TNF- $\alpha$ decreased from $25.61 \pm 12.28$ to $22.82 \pm 22.83, \mathrm{P}=0.006$ ), whereas the levels of these pro-inflammatory factors increased in hemodialysis patients without vitamin $\mathrm{C}$ supplementation (hs-CRP increased from $7.13 \pm 3.56$ to $7.73 \pm 3.83, \mathrm{P}=0.049$ and TNF- $\alpha$ increased from $21.32 \pm 5.64$ to $23.63 \pm 15.59, \mathrm{P}=0.353$ ). Vitamin $\mathrm{C}$ supplementation also reduced ESR level and increased $\mathrm{Hb}$ level significantly but it had no influence on lipid profile. 
Conclusion: According to the findings of this study, it seems that vitamin $\mathrm{C}$ administration can reduce hs-CRP and TNF- $\alpha$ level in hemodialysis patients and as a result prevent atherosclerosis. It can be concluded that administration of vitamin $\mathrm{C}$ supplementation is beneficial in hemodialysis patients.

Key words: hemodialysis, hs-CRP, TNF- $\alpha$, Vitamin C

\section{Introduction:}

Coronary artery diseases (CAD) are major causes of mortality and morbidity in chronic kidney disease and end stage renal disease patients [1] e.g. the risk of death due to CAD in 40 year-old patients is 100 times more than similar healthy patients [2]. Several studies suggested that oxidative stress and inflammation play a key role in accelerating atherosclerosis and impairment of normal endothelial function [1]. Researches showed that normal antioxidant defense mechanism in hemodialysis patients is weakened due to impairment in various components of this mechanism in un-supplemented HD patients. Vitamin $\mathrm{C}$ is one of the most important antioxidant present in human plasma. This watersoluble antioxidant is effective against lipid peroxidation, and also has hypochlorous acidscavenging ability [3]. Vitamin $\mathrm{C}$ deficiency in dialysis patients is primarily due to dietary restriction of fresh fruits and vegetables to avoid hyperkalaemia, and loss of the vitamin during dialysis sessions [4]. Data suggest that the observed deficiency of vitamin C in HD patients is not only quantitative but also qualitative. Currently, $1-1.5 \mathrm{~g}$ of oral ascorbate/week, or $300 \mathrm{mg}$ of parenteral ascorbate per dialysis session is recommended to compensate for subclinical deficiency, although evidence for such recommendations is rare [4]. It seems that, the effects of parenteral vitamin $\mathrm{C}$ supplementation on oxidative stress markers have not yet been evaluated. C-reactive protein (CRP) is one of the most sensitive inflammatory markers and it is proven that its plasma level is directly related to cardiovascular disease (CVD) in both pre-dialysis and dialysis patients. The CRP level in these patients is high (usually more than $8-10 \mathrm{mg} / \mathrm{L}$ ) in comparison with normal persons (less than $2 \mathrm{mg} / \mathrm{L}$ ). Also CRP is an inflammatory marker but also has pro-inflammatory effects and can activate the complementary system which leads to coagulation [5-8]. Although its mechanism has not been known yet, TNF- $\alpha$, one of the pro-inflammatory cytokines impairs normal endothelial function. The researchers report TNF- $\alpha$ level increases in ESRD patients [9]. So it seems that reduction of these two markers (i.e. CRP and TNF- $\alpha$ ) is a predictor of inflammation decrease in HD patients and may improve the prognosis of these patients. In this study, we intended to evaluate the effect of parentral vitamin $C$ supplementation on these markers.

\section{Methods:}

In this prospective, randomized clinical trial, 90 patients were selected randomly considering inclusion and exclusion criteria. These criteria's were as follow:

1. Adult hemodialysis patients

2. Patients who were on haemodialysis for at least 6 months.

3. Patients who had not active infectious and or inflammatory disease 
This trial was approved by the local ethics committee and was conducted in accordance with the Declaration of Helsinki. After receiving a written informed consent, patients were randomly assigned to receive, or not to receive, intravenous (IV) vitamin C (Laroscorbine, Roche Nicholas SA, Gaillard, France), $500 \mathrm{mg}$ two times per week after each dialysis session for 2 months. The control group received placebo. Blood samples were obtained at morning before beginning of dialysis. The samples witch gathered for evaluation of base hs-CRP, TNF- $\alpha$ were freezed at $-70^{\circ} \mathrm{C}$ and other routine parameters including $\mathrm{CBC}$ electrolyte, lipids levels and iron status were measured in the Imam Reza hospital laboratory. At the end of the study another blood sample was obtained to track the changes of hs-CRP, TNF- $\alpha$ and other laboratory data. hs-CRP level was measured by Monobind Inc, Lake forest, California, USA, and TNF- $\alpha$ level was measured by Human INF-alfa ELISA, Bender medsystems GmbH, Campus Vienna Biocenter, and Vienna, Austria. Other laboratory parameters were also measured by methods with international standards.

\section{Statistical analysis:}

Statistical analyses were performed using SPSS (version 16.0) package. The statistical analysis was calculated by means of $\mathrm{X}^{2}$, ANOVA and Paired samples T-test. Results have been expressed as means \pm SD. A p-value of $<0.05$ is considered as statistically significant.

\section{Results:}

Out of 90 cases only 82 patients (41 patients in each group) fulfilled inclusion and exclusion criteria and agreed to participate in the study. The main cause of exclusion of the other 8 patients was kidney transplantation $(n=2)$, malignancy $(n=1)$, hospitalization $(n=1)$ and refusal from study for personal reasons $(n=4)$. Both groups, with and without vitamin $C$ supplementation, were similar concerning age and sex ratio (Table 1).

Table 1: Comparison of Demographic Data in Vitamin C Supplemented and Control Group

\begin{tabular}{|l|l|l|l|}
\hline & With vitamin C & Without vitamin C & P \\
\hline Age (mean) & $52.54 \pm 16.17$ & $55.00+/-17.08$ & 0.504 \\
\hline Sex (male) & $58.5 \%$ & $68.3 \%$ & 0.246 \\
\hline
\end{tabular}

The mean age of patients was $53.77 \pm 16.65$ years. Causes responsible for renal failure in our patients were as follows: unknown reason $(n=28)$, hypertension $(n=18)$, diabetes mellitus $(n=13)$, glumerolonephritis $(n=11)$, polycystic kidney disease $(n=8)$ and urologic complications $(n=4)$. The mean level of hs- CRP was $7.27 \pm 3.70$ before vitamin $C$ administration and reduced to $6.6 \pm 3.75$ at the end of the study that showed significant reduction $(\mathrm{P}=0.001)$. The mean levels of $\mathrm{TNF}-\alpha$ were also $25.61 \pm 12.28$ and $22.82 \pm 22.83$ before and after vitamin supplementation respectively, which showed a significant reduction $(\mathrm{P}=0.006)$. In contrast, the mean level of hs-CRP increased significantly in control group patients $(\mathrm{P}=0.049)$ and the mean level of TNF $-\alpha$ was also non-significantly increased at the end of the study. The mean serum ferritin levels were high $(>500 \mathrm{ng} / \mathrm{ml})$ in both groups. However, there was no significant reduction in serum ferritin level during the study. Table 2 shows the other findings of this study. 
Table 2: Comparison of Laboratory Data between Vitamin C Supplemented and Control Group Patients on Hemodialysis

\begin{tabular}{|l|l|l|l|l|l|l|}
\hline & \multicolumn{3}{|c|}{ With vitamin C } & \multicolumn{3}{c|}{ Without vitamin C } \\
\hline & Phase 1 & Phase 2 & $\mathrm{p}$ & Phase 1 & Phase 2 & $\mathrm{p}$ \\
\hline $\mathrm{Hb}$ & $10.25 \pm 1.81$ & $10.76 \pm 1.90$ & 0.022 & $10.33 \pm 2.36$ & $10.73 \pm 2.01$ & 0.138 \\
\hline Hct & $32.69 \pm 5.89$ & $33.98 \pm 5.96$ & 0.067 & $33.52 \pm 7.30$ & $33.82 \pm 6.22$ & 0.716 \\
\hline Cholesterol & $177.83 \pm 46.62$ & $166.00 \pm 43.72$ & 0.882 & $161.54 \pm 55.95$ & $161.85 \pm 43.31$ & 0.967 \\
\hline TG & $174.80 \pm 101.23$ & $176.90 \pm 95.36$ & 0.882 & $158.22 \pm 80.73$ & $157.80 \pm 90.08$ & 0.967 \\
\hline ALP & $408.20 \pm 181.31$ & $464.22 \pm 309.86$ & 0.186 & $366.00 \pm 192.52$ & $459.56 \pm 415.34$ & 0.099 \\
\hline Albumin & $4.06 \pm 0.49$ & $4.12 \pm 0.48$ & 0.394 & $3.84 \pm 0.59$ & $4.04 \pm 0.50$ & 0.080 \\
\hline Serum Iron & $83.78 \pm 37.65$ & $88.34 \pm 47.67$ & 0.541 & $74.44 \pm 41.81$ & $64.46 \pm 26.09$ & 0.144 \\
\hline TIBC & $237.59 \pm 52.79$ & $245.24 \pm 65.71$ & 0.225 & $253.15 \pm 69.28$ & $225.20 \pm 45.92$ & 0.508 \\
\hline Ferritin & $658.83 \pm 376.89$ & $882.07 \pm 784.75$ & 0.050 & $613.22 \pm 399.93$ & $696.66 \pm 471.92$ & 0.191 \\
\hline ESR & $41.76 \pm 27.01$ & $34.07 \pm 20.08$ & 0.041 & $41.66 \pm 26.68$ & $39.00 \pm 30.10$ & 0.356 \\
\hline
\end{tabular}

\section{Discussion:}

It has been proved that pro inflammatory factors increase the risk of cardiovascular diseases and mortality especially in hemodialysis patients. Nutritional supplementation such as vitamin $\mathrm{C}$ may become an additional therapy to reduce these factors and improve outcomes in hemodialysis patients [10]. Available recent evidence suggests that certain nutrients and/or antioxidants may have a significant modulatory role on cytokine biology, which is of much interest as advanced oxidation products may be mediators of inflammation in ESRD patients. Supplementation with vitamin E also decreases CRP and monocyte IL-6 levels in non-renal patient groups. Thus, as vitamin $\mathrm{E}$ has been shown to decrease the oxidative susceptibility of low-density lipoprotein and to reduce cardiovascular end points in HD patients, it would be of interest to study the impact of vitamin E on inflammatory parameters in ESRD patients [11-13]. A wide range of CRP levels in CRF patients are reported in the literature. Docci et al. observed CRP levels of $7.4 \mathrm{mg} / \mathrm{dl}$ in dialysis patients, and Zimmermann et al. reported that the increase in the CRP levels was $46 \%$ in hemodialysis patients [14-16]. Owen and Lowrie [17] reported that this increase was $35 \%$. In our study, in the group with no vitamin $\mathrm{C}$ supplementation CRP level was 7.13 and increased $1 \%$ in time. There are limited data regarding the effects of vitamin $\mathrm{C}$ supplementation on inflammation status in dialysis patients. In non-renal patients, vitamin C supplementation can decrease CRP [18]. According to the results of this study, intravenous administration of vitamin $\mathrm{C}$ can significantly reduce plasma inflammatory markers i.e. Hs-CRP and TNF- $\alpha$, whereas in patients without vitamin C supplementation there was a significant rise in plasma inflammatory markers mentioned above. This rise in the latter group could be due to the time elapsed during this study which shows the deterioration of patients' situation by time in case of lack of vitamin $\mathrm{C}$ supplementation. This finding can be a result of inhibition of baneful inflammatory mechanism that is responsible for impaired endothelial function in HD patients. A prior study by Fumeron et al showed that administration of oral Vitamin $\mathrm{C}$ with similar doses had no beneficial effect on reducing inflammatory markers [6]. In another study, Keven and coworkers failed to observe a significant effect of a 6-month vitamin $\mathrm{C}$ supplementation on 
CRP and albumin levels [19]. Probably this finding is a result of better bioavailability of intravenous vitamin $\mathrm{C}$ which leads to a higher level of vitamin $\mathrm{C}$ concentration in plasma and reduction in inflammatory response. Different ways of vitamin $\mathrm{C}$ supplementation (oral versus intravenous) may be the cause of different findings in Fumeron and our study. Also, the racial differences between our study and Fumeron study may play a role in this difference $[6,21]$. In this study, mean $\mathrm{Hb}$ level increased significantly in vitamin C-treated patients which is compatible with past studies [22]. The increase in Hb levels may be due to reduction of inflammation that overcomes erythropoietin resistance in these patients [23]. Ascorbic Acid, as an antioxidant, can increase iron release of ferritin and the reticoloendothelial system accelerates available iron. Therefore, vitamin $\mathrm{C}$ may reduce functional iron deficiency of dialysis patients [24]. The serum level of ferritin was $<500 \mathrm{ng} / \mathrm{ml}$ in this study. Vitamin C administration did not affect the serum ferritin levels. It is not clear how much ferritin level is needed to increase the effect of vitamin $\mathrm{C}$ on improvement of functional iron deficiency of dialysis patients. One of the limitations of our study was the absence of baseline serum level of vitamin $\mathrm{C}$ in our dialysis patients.

Conclusion: It seems that short-term IV vitamin $\mathrm{C}$ supplementation can modify well-defined inflammation markers and also improve anemia in HD patients. On the whole, administration of vitamin $\mathrm{C}$ supplementation is recommended in hemodialysis patients. We need more studies to evaluation of the effects vitamin $\mathrm{C}$ on dialysis patients.

Acknowledgements: The authors thank the Leila Razmjou, staff and the nurses of Tabriz university hospitals for their efficient and kind collaboration.

\section{Competing interests:}

The authors declare that there are no competing interests.

Author's Contributions: All the authors contributed equally.

Abbreviations: Intravenously (IV), Coronary artery diseases (CAD), End Stage Renal Disease patients (ESRD), Hemodialysis patients (HD patients), Tumor Necrosis Factor- $\alpha$ (TNF- $\alpha$ ), C-reactive protein (CRP), and Cardiovascular disease (CVD)

\section{References:}

1. Go AS, Chertow GM, Fan D, et al. Chronic kidney disease and the risks of death, cardiovascular events, and hospitalization. N Engl J Med; 2004. 351: 1296-1305.

2. Ridker PM, Cushman M, Stampfer MJ, Tracy RP, Hennekens CH. Plasma concentration of C-reactive protein and risk of developing peripheral vascular disease. Circulation; 1998. 97: 425-8.

3. Ridker PM, Cushman M, Stampfer MJ, Tracy RP, Hennekens CH. Inflammation, aspirin, and the risk of cardiovascular disease in apparently healthy men. $N$ Engl $J$ Med; 1997. 336: 973-9.

4. Haverkate F, Thompson SG, Pyke SD, Gallimore JR, Pepys MB. Production of Creactive protein and risk of coronary events in stable and unstable angina. European 
Concerted Action on Thrombosis and Disabilities Angina Pectoris Study Group. Lancet; 1997. 349: 462-6.

5. Stenvinkel P, Alvestrand A. Inflammation in End-stage Renal Disease: Sources, Consequences, and Therapy. Seminars in Dialysis; 2002. 15: 329-337.

6. Fumeron C, Nguyen-Khoa T, Saltiel C, Kebede M, Buisson C, Drueke TB. Effects of oral vitamin $\mathrm{C}$ supplementation on oxidative stress and inflammation status in hemodialysis patients. Nephrol Dial Transplant; 2005. 20: 1874-1879.

7. Boenisch O, Ehmke KD, Heddergott A, Naoum C, Frei U, Schindler R. C-reactiveprotein and cytokine plasma levels in hemodialysis patients. J Nephrol; 2002. 15: 547-551.

8. Prasad K. C-Reactive Protein (CRP)-Lowering Agents. Cardiovascular Drug Reviews; 2006. 24: 33-50.

9. Stenvinkel P. Endothelial dysfunction and inflammation-is there a link. Nephrol Dial Transplant; 2001. 16: 1968-1971.

10. Kalantar-Zadeh $\mathrm{K}$ et al. An anti-inflammatory and antioxidant nutritional supplement for hypoalbuminemic HD patients: A pilot/feasibility study. Jrnl of Renal Nutr; 2005. 15(3): 318-31.

11. Stenvinkel P. Inflammation in end-stage renal failure: could it be treated?" Nephrol Dial Transplant; 2002. 17(8): 33-38.

12. Witko-Sarsat V, Friedlander M, Khoa TN, Capeille're-Blandin C, Nguyen AT, Canteloup S, et al. Advanced oxidation protein products as novel mediators of inflammation andmonocyte activation in chronic renal failure. J Immunol; 1998. 161: 2524-2532.

13. Grimble RF. Nutritional modulation of cytokine biology. Nutrition; 1998. 14: 634640.

14. Borazan A, Ustun H, Ustundag Y, Aydemir S, Bayraktaroglu T, Sert M, et al. The effects of peritoneal dialysis and hemodialysis on serum tumor necrosis factor-alpha, interleukin-6, interleukin-10 and C-reactive-protein levels. Mediators of Inflammation; 2004. 13(3): 201-204.

15. Docci D, Bilancioni R, Buscaroli A, et al. Elevated serum levels of C-reactive protein in haemodialysis patients. Nephron; 1990. 56: 364-367.

16. Zimmermann J, Herrlinger S, Pruy A, Metzger T, Wanner C. Inflammation enhances cardiovascular risk and mortality in haemodialysis. Kidney Int; 1999. 55: 648-658.

17. Owen WF, Lowrie EG. C-reactive protein as an outcome predictor for maintenance haemodialysis patients. Kidney Int; 1998. 54: 627-636.

18. Block G, Jensen CD, Dalvi TB, Norkus EP, Hudes M, Crawford PB, et al. Vitamin C treatment reduces elevated C-reactive protein. Free Radical Biology \& Medicine; 2009. 46: 70-77.

19. Keven K, Kutlay S, Nergizoglu G, Erturk S. Randomized, crossover study of the effect of vitamin C on EPO response in hemodialysis patients. Am J Kidney Dis; 2003. 41: 1233-1239.

20. Padayatty SJ, Sun H, Wang Y et al. Vitamin C pharmacokinetics: implications for oral and intravenous use. Ann Intern Med; 2004. 140: 533-537. 
21. Mezzano D, Pais E.O, Aranda E, Panes O, Downey P, Ortis M. Inflammation, not hyperhomocysteinemia, is related to oxidative stress and hemostatic and endothelial dysfunction in uremia. Kidney Internationa; 2001. 60: 1844-1850.

22. Steveninkel P, Bareny P. Anemia, rHuEPO resistance, and cardiovascular disease in end stage renal failure: link to inflammation and oxidative stress. Nephrol Dial Transplant; 2002.17 (5), 32-37.

23. Espahbodi F, Kashi Z, Ala S, Hendoii N. Efficacy and Safety of Oral Versus Intravenous

24. Vitamin C in Hemodialysis Patients with Functional Iron Deficiency. Int J Endocrinol Metab; 2007. 3 129-134. 